University of Nebraska - Lincoln

DigitalCommons@University of Nebraska - Lincoln

Faculty Publications: Materials Research

Science and Engineering Center

Materials Research Science and Engineering

Center

5-1-2005

\title{
A symmetric Green function for the non-collinear magnetic multilayer
}

Mikhail Ye Zhuravlev

University of Nebraska - Lincoln, myezhur@gmail.com

John D. Burton

University of Nebraska-Lincoln, jburton2@unl.edu

A. V. Vedyayev

M. V. Lomonosov Moscow State University

Evgeny Y. Tsymbal

University of Nebraska-Lincoln, tsymbal@unl.edu

Follow this and additional works at: https://digitalcommons.unl.edu/mrsecfacpubs

Part of the Materials Science and Engineering Commons

Zhuravlev, Mikhail Ye; Burton, John D.; Vedyayev, A. V.; and Tsymbal, Evgeny Y., "A symmetric Green function for the non-collinear magnetic multilayer" (2005). Faculty Publications: Materials Research Science and Engineering Center. 30.

https://digitalcommons.unl.edu/mrsecfacpubs/30

This Article is brought to you for free and open access by the Materials Research Science and Engineering Center at DigitalCommons@University of Nebraska - Lincoln. It has been accepted for inclusion in Faculty Publications: Materials Research Science and Engineering Center by an authorized administrator of DigitalCommons@University of Nebraska - Lincoln. 
Published in Journal Of Physics A: Mathematical And General 38 (17 June 2005), pp. 5547-5556. doi:10.1088/0305-4470/38/24/008 Online at http://stacks.iop.org/JPhysA/38/5547

Copyright (C 2005 Institute Of Physics Publishing. Used by permission.

\title{
A symmetric Green function for the non-collinear magnetic multilayer
}

\author{
M. Ye. Zhuravlev*, J. D. Burton*, A. V. Vedyayev†, and E. Y. Tsymbal* \\ * Department of Physics and Astronomy, Center for Materials Research \\ and Analysis, University of Nebraska-Lincoln \\ $\dagger$ Department of Physics, M. V. Lomonosov Moscow State University
}

Submitted January 2005, revised January 2005, published 1 June 2005

\begin{abstract}
Symmetry properties of the Green function in magnetic multilayers with noncollinear magnetization of the layers are investigated on the basis of the transfer matrix method. The Green function symmetric with respect to permutation of its arguments is constructed. It is shown how the boundary conditions can be imposed on this Green function.
\end{abstract}

\section{Introduction}

Magnetic multilayered structures demonstrating giant magnetoresistance (GMR) [1] and tunnelling magnetoresistance (TMR) [2] attract great attention due to their potential applications and interesting physics. A fairly simple free-electron-like model is a promising tool for investigating new phenomena in these structures [3-7]. In particular, transport properties of the layered structures have attracted considerable attention. One of the frequently used approaches to the calculation of transport properties is the Kubo linear response formalism, which requires the construction of the Green function (GF) of the multilayer.

In systems with complicated geometry, the construction of the GF is a cumbersome task. The one-electron GF must obey the Schrödinger equation with a delta-function source term. The continuity condition on the GF and its first derivative at the interfaces must be fulfilled. In addition, the geometry of the system implies some boundary conditions for the GF. On the other hand, it is convenient to build the GF in such a way that it has certain symmetry properties with respect to permutation of its arguments. The latter problem, therefore, has to be solved for the GF, which is continuous at the interfaces and obeys the boundary conditions In the one-dimensional case, the GF can be chosen symmetric with respect to permutation of its arguments: $G\left(z, z^{\prime}\right)=G\left(z^{\prime}, z\right)$. However, as was noted in [8], this permutation symmetry is not, in general, a property of the GF. Therefore, for each particular case the problem of GF symmetry requires additional investigation. 
In a multilayered system with non-collinear magnetization, the GF is a $4 \times 4$ matrix [9]. A way to construct the GF of a magnetic multilayer with non-collinear magnetization of the layers was proposed in [10], using the so-called $k_{\|}-z$ representation. The $k_{\|}-z$ representation takes the advantage of the $k$-space representation in the plane of the multilayer $(k \|$ is the transverse wave vector), but uses a real space representation along the $z$-axis perpendicular to the plane of the layers. The $k_{\|}-z$ representation is especially convenient for the investigation of transport properties. Special attention in [10] was paid to the matching of the GF at the interfaces.

In this paper, we investigate the symmetry of the GF with respect to permutation of its arguments for magnetic multilayerswith non-collinear magnetization using the transfer-matrix method. We demonstrate how the boundary conditions can be imposed on this Green function. The proposed method is a generalization of the well-known method of constructing the GF for the second-order linear differential equation

$$
y^{\prime \prime}(z)+q(z) y(z)=0
$$

Using two linearly independent solutions $f 1(z), f 2(z)$ of this equation, the GF is given by

$$
G\left(z, z^{\prime}\right)=\frac{1}{W\left(f_{1}, f_{2}\right)} \begin{cases}f_{1}(z) f_{2}\left(z^{\prime}\right), & z>z^{\prime} \\ f_{1}\left(z^{\prime}\right) f_{2}(z), & z<z^{\prime}\end{cases}
$$

where the Wronskian $W\left(f_{1}, f_{2}\right) \equiv f_{1}^{\prime}(z) f_{2}(z)-f_{1}(z) f_{2}^{\prime}(z)$ does not depend on $z$. The GF obeys the equation

$$
\frac{\partial^{2} G\left(z, z^{\prime}\right)}{\partial z^{2}}+q(z) G\left(z, z^{\prime}\right)=\delta\left(z-z^{\prime}\right) .
$$

This method is different from that proposed in [11], where the problem of surface Green function matching was solved for an arbitrary number of interfaces.

\section{Construction of the Green function}

The Green function for amagnetic multilayer system with non-collinear magnetization in each layer satisfies the following second-order ordinary differential equation [9]:

$$
\begin{array}{r}
\frac{\hbar^{2}}{2 m}\left[\left(\frac{\partial^{2}}{\partial z^{2}}+\left(k_{F}^{(n)}\right)^{2}-k_{\|}^{2}-E_{0}^{(n)}\right)\left(\begin{array}{cc}
1 & 0 \\
0 & 1
\end{array}\right)-E_{1}^{(n)}\left(\begin{array}{cc}
\cos \theta_{n} & \sin \theta_{n} \\
\sin \theta_{n} & -\cos \theta_{n}
\end{array}\right)\right] \\
\times\left(\begin{array}{ll}
G_{n m}^{\uparrow \uparrow}\left(z, z^{\prime}\right) & G_{n m}^{\uparrow \downarrow}\left(z, z^{\prime}\right) \\
G_{n m}^{\downarrow \uparrow}\left(z, z^{\prime}\right) & G_{n m}^{\downarrow \downarrow}\left(z, z^{\prime}\right)
\end{array}\right)=\left(\begin{array}{cc}
\delta\left(z-z^{\prime}\right) & 0 \\
0 & \delta\left(z-z^{\prime}\right)
\end{array}\right),
\end{array}
$$

where $n$ indexes the layer in which $z$ lies, $m$ indexes the layer in which $z^{\prime}$ lies and $k_{\|}$is an inplane wave vector. $\theta_{n}$ is the angle between the $x$-axis (which is perpendicular to the $z$-axis) and the magnetization within the $n$th layer. For non-magnetic layers, the value of $\theta_{n}$ can be chosen arbitrarily. $E_{0}^{(n)}$ and $E_{1}^{(n)}$ determine the position of the bottom of the band in the $n$th layer and the exchange splitting, respectively. For non-magnetic layers $E_{1}^{(n)}=0$. In the following we will give the GF in units of $\frac{\hbar^{2}}{2 m}$ (i.e., put $\frac{\hbar^{2}}{2 m}=1$ ). We consider a multilayer whose layers are numbered 0 to $N$ from right to left with interfaces at $z=c_{1}, c_{2}, \ldots, c_{N}$.

If we define

$$
\mathbf{G}_{n m}^{\uparrow}\left(z, z^{\prime}\right) \equiv\left(\begin{array}{c}
G_{n m}^{\uparrow \uparrow}\left(z, z^{\prime}\right) \\
W_{n m}^{\uparrow \uparrow}\left(z, z^{\prime}\right) \\
G_{n m}^{\downarrow \uparrow}\left(z, z^{\prime}\right) \\
W_{n m}^{\downarrow \uparrow}\left(z, z^{\prime}\right)
\end{array}\right)=\left(\begin{array}{c}
G_{n m}^{\uparrow \uparrow}\left(z, z^{\prime}\right) \\
\frac{\partial}{\partial z} G_{n m}^{\uparrow \uparrow}\left(z, z^{\prime}\right) \\
G_{n m}^{\downarrow \uparrow}\left(z, z^{\prime}\right) \\
\frac{\partial}{\partial z} G_{n m}^{\downarrow \uparrow}\left(z, z^{\prime}\right)
\end{array}\right)
$$


and

$$
\mathbf{G}_{n m}^{\downarrow}\left(z, z^{\prime}\right) \equiv\left(\begin{array}{c}
G_{n m}^{\uparrow \downarrow}\left(z, z^{\prime}\right) \\
W_{n m}^{\uparrow \downarrow}\left(z, z^{\prime}\right) \\
G_{n m}^{\downarrow \downarrow}\left(z, z^{\prime}\right) \\
W_{n m}^{\downarrow \downarrow}\left(z, z^{\prime}\right)
\end{array}\right)=\left(\begin{array}{c}
G_{n m}^{\uparrow \downarrow}\left(z, z^{\prime}\right) \\
\frac{\partial}{\partial z} G_{n m}^{\uparrow \downarrow}\left(z, z^{\prime}\right) \\
G_{n m}^{\downarrow \downarrow}\left(z, z^{\prime}\right) \\
\frac{\partial}{\partial z} G_{n m}^{\downarrow \downarrow}\left(z, z^{\prime}\right)
\end{array}\right)
$$

then we can write equation (4) as two systems of four first-order ordinary differential equations:

$$
\left(\mathbf{I} \frac{\partial}{\partial z}+\mathbf{L}_{n}\right) \mathbf{G}_{n m}^{\uparrow}\left(z, z^{\prime}\right)=\left(\begin{array}{c}
0 \\
\delta\left(z-z^{\prime}\right) \\
0 \\
0
\end{array}\right)
$$

and

$$
\left(\mathbf{I} \frac{\partial}{\partial z}+\mathbf{L}_{n}\right) \mathbf{G}_{n m}^{\downarrow}\left(z, z^{\prime}\right)=\left(\begin{array}{c}
0 \\
0 \\
0 \\
\delta\left(z-z^{\prime}\right)
\end{array}\right) \text {. }
$$

Here we have defined

$$
\mathbf{L}_{n} \equiv\left(\begin{array}{cccc}
0 & -1 & 0 & 0 \\
\left(k_{F}^{(n)}\right)^{2}-k_{\|}^{2}-E_{0}^{(n)}-E_{1}^{(n)} \cos \theta_{n} & 0 & -E_{1}^{(n)} \sin \theta_{n} & 0 \\
0 & 0 & 0 & -1 \\
-E_{1}^{(n)} \sin \theta_{n} & 0 & \left(k_{F}^{(n)}\right)^{2}-k_{\|}^{2}-E_{0}^{(n)}+E_{1}^{(n)} \cos \theta_{n} & 0
\end{array}\right)
$$

Note that both equations (7) and (8) differ only on the right-hand side.

Recall that for a system of the form

$$
\frac{\mathrm{d} \Psi(z)}{\mathrm{d} z}+\mathbf{L} \Psi(z)=\mathbf{f}(z)
$$

the solution can be expressed in terms of a fundamental matrix $\mathbf{F}$ as follows:

$$
\Psi(z)=\mathbf{F}(z) \int_{0}^{z} \mathbf{F}^{-1}(s) \mathbf{f}(s) \mathrm{d} s+\mathbf{F}(z) \mathbf{h},
$$

where $\mathbf{h}$ is a column. Equations (7) and (8) constitute two $4 \times 4$ systems of the form (10) with

$$
\mathbf{f}^{\uparrow}\left(z, z^{\prime}\right)=\left(\begin{array}{c}
0 \\
\delta\left(z-z^{\prime}\right) \\
0 \\
0
\end{array}\right), \quad \mathbf{f}^{\downarrow}\left(z, z^{\prime}\right)=\left(\begin{array}{c}
0 \\
0 \\
0 \\
\delta\left(z-z^{\prime}\right)
\end{array}\right)
$$

Thus, we can write the Green function solution in the form

$$
\mathbf{G}\left(z, z^{\prime}\right)=\mathbf{F}(z) \int_{0}^{z}(\mathbf{F}(s))^{-1} \mathbf{f}\left(s, z^{\prime}\right) \mathrm{d} s+\mathbf{F}(z) \mathbf{h}\left(z^{\prime}\right),
$$

where we choose $\mathbf{h}\left(z^{\prime}\right)$ so that the Green function obeys the boundary conditions and has the proper symmetry. 
To find a fundamental matrix for equations (7) and (8), we must first find a solution to the corresponding homogeneous equation

$$
\left(\mathbf{I} \frac{\partial}{\partial z}+\mathbf{L}_{n}\right) \Phi^{(n)}(z)=\left(\begin{array}{l}
0 \\
0 \\
0 \\
0
\end{array}\right) .
$$

There are four linearly independent solutions of equation (14). We can arrange these solutions as the columns of a fundamental matrix for the operator $\mathbf{I} \frac{\partial}{\partial z}+\mathbf{L}_{n}$. It can be easily seen by using the ansatz

$$
\varphi=\left(\begin{array}{c}
A_{1} \mathrm{e}^{\mathrm{i} k z} \\
\mathrm{i} k A_{1} \mathrm{e}^{\mathrm{i} k z} \\
A_{2} \mathrm{e}^{\mathrm{i} k z} \\
\mathrm{i} k A_{2} \mathrm{e}^{\mathrm{i} k z}
\end{array}\right),
$$

that a fundamental matrix is given by

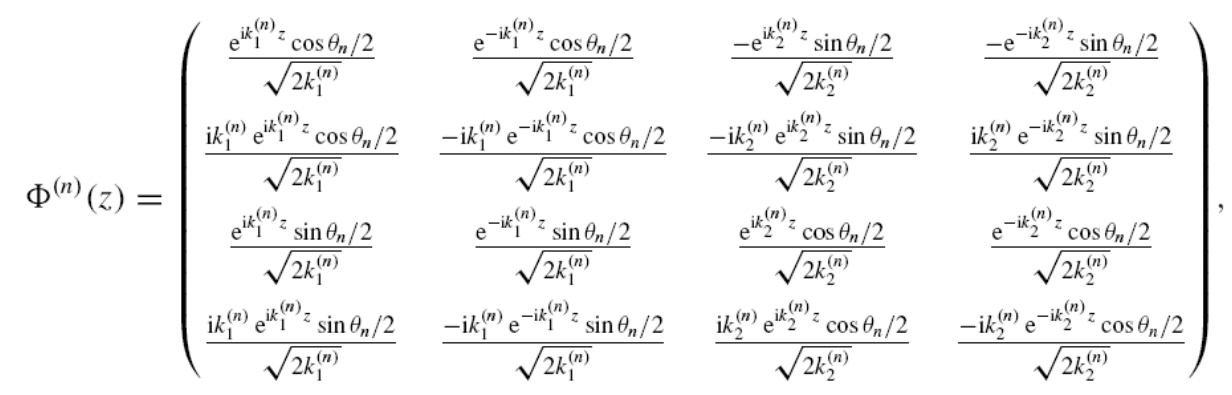

where

$$
k_{1,2}^{(n)}=\sqrt{\frac{2 m E_{F}}{\hbar^{2}}-k_{\|}^{2}-\frac{2 m}{\hbar^{2}}\left(E_{0}^{(n)} \pm E_{1}^{(n)}\right)} .
$$

Note that these solutions are defined for each layer and generally do not match at the interfaces. Here we introduce a set of matrices $\mathbf{F}^{(n)}(z)$ that do match at the interfaces. Any other solution can be represented as a linear combination of the columns of equation (16), i.e. $\mathbf{F}^{(n)}(z)=\Phi(n)(z) \mathbf{A}_{n}$. Then at the $n$th interface the matching of the $\mathbf{F}^{(n)}(z)$ functions means

$$
\Phi^{(n-1)}\left(c_{n}\right) \mathbf{A}_{n-1}=\Phi^{(n)}\left(c_{n}\right) \mathbf{A}_{n} .
$$

Thus the matrices $\mathbf{A}_{n}$ are related by

$$
\mathbf{A}_{n}=\left(\Phi^{(n)}\left(c_{n}\right)\right)^{-1} \Phi^{(n-1)}\left(c_{n-1}\right) \mathbf{A}_{n-1} .
$$

Clearly the $\mathbf{F}^{(n)}(z)$ constitutes a fundamental matrix for the operator in equations (7) and (8) provided $\operatorname{det}\left[A^{(n)}\right] \neq 0$.

The GF of the form (13) obeys equations (7) and (8) if

and

$$
\mathbf{G}^{\uparrow}\left(z, z^{\prime}\right)= \begin{cases}\mathbf{F}(z)\left(\mathbf{F}_{2}^{-1}\left(z^{\prime}\right)+\mathbf{h}^{\uparrow}\left(z^{\prime}\right)\right), & z>z^{\prime} \\ \mathbf{F}(z) \mathbf{h}^{\uparrow}\left(z^{\prime}\right), & z<z^{\prime}\end{cases}
$$

$$
\mathbf{G}^{\downarrow}\left(z, z^{\prime}\right)=\left\{\begin{array}{ll}
\mathbf{F}(z)\left(\mathbf{F}_{4}^{-1}\left(z^{\prime}\right)+\mathbf{h}^{\downarrow}\left(z^{\prime}\right)\right), & z>z^{\prime} \\
\mathbf{F}(z) \mathbf{h}^{\downarrow}\left(z^{\prime}\right), & z<z^{\prime}
\end{array} .\right.
$$

Here we have written $\mathbf{F}_{n}^{-1}\left(z^{\prime}\right)$ for the $n$th column of the inverse matrix $\mathbf{F}^{-1}$ evaluated at $z^{\prime}$. 
Now we attempt to write equation (20) in terms of the basic functions given in equation (16). Suppose that $z$ is in the $n$th layer and that $z^{\prime}$ is in the $m$ th layer. Then we obtain, for $z>z^{\prime}$,

$$
\begin{aligned}
\mathbf{G}^{\uparrow}\left(z>z^{\prime}\right) & =\mathbf{F}(z)\left(\mathbf{F}_{2}^{-1}\left(z^{\prime}\right)+\mathbf{h}^{\uparrow}\left(z^{\prime}\right)\right) \\
& =\Phi^{(n)}(z) \mathbf{A}^{(n)}\left(\left(\Phi^{(m)}\left(z^{\prime}\right) \mathbf{A}^{(m)}\right)_{2}^{-1}+\mathbf{h}^{\uparrow}\left(z^{\prime}\right)\right) \\
& =\Phi^{(n)}(z)\left(\mathbf{A}^{(n)}\left(\mathbf{A}^{(m)}\right)^{-1}\right)\left(\left(\Phi^{(m)}\left(z^{\prime}\right)\right)_{2}^{-1}+\mathbf{A}^{(m)} \mathbf{h}^{\uparrow}\left(z^{\prime}\right)\right) \\
& =\Phi^{(n)}(z) \mathbf{A}^{(n)}\left(\mathbf{A}^{(m)}\right)^{-1}\left(\mathbf{I}+\mathbf{X}^{(m)}\right)\left(\Phi^{(m)}\left(z^{\prime}\right)\right)_{2}^{-1}
\end{aligned}
$$

Here we mean for $\left(\Phi^{(m)}\left(z^{\prime}\right)\right)_{p}^{-1}$ the $p$ th column of the inverse of $\Phi^{(m)}\left(z^{\prime}\right)$. In equation (22) we postulated that $\mathbf{A}^{(m)} \mathbf{h}^{\uparrow}\left(z^{\prime}\right)$ can be written as $\mathbf{X}^{(m)}\left(\Phi^{(m)}\left(z^{\prime}\right)\right)_{2}^{-1}$, where $\mathbf{X}^{(m)}$ is a matrix that is determined by the boundary and symmetry conditions. Using the same considerations for both branches of equations (20) and (21) we can write the GF as follows:

$$
\mathbf{G}^{\uparrow}\left(z, z^{\prime}\right)= \begin{cases}\Phi^{(n)}(z) \mathbf{A}^{(n)}\left(\mathbf{A}^{(m)}\right)^{-1}\left(\mathbf{I}+\mathbf{X}^{(m)}\right)\left(\Phi^{(m)}\left(z^{\prime}\right)\right)_{2}^{-1}, & z>z^{\prime} \\ \Phi^{(n)}(z) \mathbf{A}^{(n)}\left(\mathbf{A}^{(m)}\right)^{-1} \mathbf{X}^{(m)}\left(\Phi^{(m)}\left(z^{\prime}\right)\right)_{2}^{-1}, & z<z^{\prime},\end{cases}
$$

and

$$
\mathbf{G}^{\downarrow}\left(z, z^{\prime}\right)=\left\{\begin{array}{ll}
\Phi^{(n)}(z) \mathbf{A}^{(n)}\left(\mathbf{A}^{(m)}\right)^{-1}\left(\mathbf{I}+\mathbf{Y}^{(m)}\right)\left(\Phi^{(m)}\left(z^{\prime}\right)\right)_{4}^{-1}, & z>z^{\prime} \\
\Phi^{(n)}(z) \mathbf{A}^{(n)}\left(\mathbf{A}^{(m)}\right)^{-1} \mathbf{Y}^{(m)}\left(\Phi^{(m)}\left(z^{\prime}\right)\right)_{4}^{-1}, & z<z^{\prime}
\end{array} .\right.
$$

In equation (24), $\mathbf{Y}$ plays the same role as $\mathbf{X}$ in equation (23).

If we define two auxiliary matrices

and

$$
\mathbf{P}_{1}=\left(\begin{array}{cccc}
0 & 1 & 0 & 0 \\
-1 & 0 & 0 & 0 \\
0 & 0 & 0 & 1 \\
0 & 0 & -1 & 0
\end{array}\right)
$$

$$
\mathbf{P}_{2}=\left(\begin{array}{cccc}
0 & -\mathrm{i} & 0 & 0 \\
\mathrm{i} & 0 & 0 & 0 \\
0 & 0 & 0 & -\mathrm{i} \\
0 & 0 & \mathrm{i} & 0
\end{array}\right)
$$

then it is straightforward to see that

$$
\left(\Phi^{(n)}(z)\right)^{-1}=\left(\mathbf{P}_{1} \Phi^{(n)}(z) \mathbf{P}_{2}\right)^{T}=\left(\mathbf{P}_{1}^{T} \Phi^{(n)}(z) \mathbf{P}_{2}^{T}\right)^{T} .
$$

It is then a simple exercise to show that

$$
\begin{aligned}
& \left(\Phi^{(m)}\left(z^{\prime}\right)\right)_{2}^{-1}=P_{2}\left(\Phi^{(m)}\left(z^{\prime}\right)\right)_{1}^{T} \\
& \left(\Phi^{(m)}\left(z^{\prime}\right)\right)_{4}^{-1}=P_{2}\left(\Phi^{(m)}\left(z^{\prime}\right)\right)_{3}^{T} .
\end{aligned}
$$

Here we mean for $\left(\Phi^{(m)}\left(z^{\prime}\right)\right)_{p}^{T}$ the $p$ th column of the transpose of $\Phi^{(m)}\left(z^{\prime}\right)$. The relations (28) show that each fundamental matrix $\Phi^{(n)}(z)$ differs from a symplectic matrix only by a constant multiplier, namely $\left(\frac{1+\mathrm{i}}{\sqrt{2}}\right)$.

\section{Symmetry of the Green function}

We are looking for a solution of equation (4) that is symmetric with respect to permutation of the arguments:

$$
G_{n m}^{\sigma \sigma^{\prime}}\left(z, z^{\prime}\right)=G_{m n}^{\sigma^{\prime} \sigma}\left(z^{\prime}, z\right)
$$


For diagonal elements of the GF this implies

$$
G_{n m}^{\uparrow \uparrow}\left(z, z^{\prime}\right)=G_{m n}^{\uparrow \uparrow}\left(z^{\prime}, z\right) \text {. }
$$

For $z>z^{\prime}$ we have $G_{n m}^{\uparrow \uparrow}\left(z>z^{\prime}\right)=G_{m n}^{\uparrow \uparrow}\left(z^{\prime}<z\right)$. Since $G^{\uparrow \uparrow}$ is the first element of equation (23) we obtain

$$
\sum_{j, k=1}^{4} \Phi_{1 j}^{(n)}(z)\left(\mathbf{T}^{(n m)}\left(\mathbf{I}+\mathbf{X}^{(m)}\right) \mathbf{P}_{2}\right)_{j k} \Phi_{1 k}^{(m)}\left(z^{\prime}\right)=\sum_{j, k=1}^{4} \Phi_{1 k}^{(m)}\left(z^{\prime}\right)\left(\mathbf{T}^{(m n)} \mathbf{X}^{(n)} \mathbf{P}_{2}\right)_{k j} \Phi_{1 j}^{(n)}(z) .
$$

Here we have denoted

$$
\mathbf{A}^{(n)}\left(\mathbf{A}^{(m)}\right)^{-1}=\mathbf{T}^{(n m)}
$$

which is known as the transfer matrix. It is easily seen from equation (31) that

$$
\mathbf{T}^{(n m)}\left(\mathbf{I}+\mathbf{X}^{(m)}\right) \mathbf{P}_{2}=\left(\mathbf{T}^{(m n)} \mathbf{X}^{(n)} \mathbf{P}_{2}\right)^{T} .
$$

An equivalent derivation for the symmetry of the $\downarrow \downarrow$ component leads to

$$
\mathbf{T}^{(n m)}\left(\mathbf{I}+\mathbf{Y}^{(m)}\right) \mathbf{P}_{2}=\left(\mathbf{T}^{(m n)} \mathbf{Y}^{(n)} \mathbf{P}_{2}\right)^{T} .
$$

For non-diagonal elements of the GF equation (29) forces

$$
G_{n m}^{\downarrow \uparrow}\left(z, z^{\prime}\right)=G_{m n}^{\uparrow \downarrow}\left(z^{\prime}, z\right) \text {. }
$$

This implies a symmetry relationship between the third element of equation (5) and the first element of equation (6). That is

$$
\sum_{j, k=1}^{4} \Phi_{3 j}^{(n)}(z)\left(\mathbf{T}^{(n m)}\left(\mathbf{I}+\mathbf{X}^{(m)}\right) \mathbf{P}_{2}\right)_{j k} \Phi_{1 k}^{(m)}\left(z^{\prime}\right)=\sum_{j, k=1}^{4} \Phi_{1 k}^{(m)}\left(z^{\prime}\right)\left(\mathbf{T}^{(m n)} \mathbf{Y}^{(n)} \mathbf{P}_{2}\right)_{k j} \Phi_{3 j}^{(n)}(z) .
$$

Thus we see that

$$
\mathbf{T}^{(n m)}\left(\mathbf{I}+\mathbf{X}^{(m)}\right) \mathbf{P}_{2}=\left(\mathbf{T}^{(m n)} \mathbf{Y}^{(n)} \mathbf{P}_{2}\right)^{T} .
$$

Comparing equations (37) and (33), we find that $\mathbf{Y}^{(n)}=\mathbf{X}^{(n)}$.

Note that using equation (19) it can be shown that

$$
\left(\mathbf{T}^{(n m)}\right)^{T}=\mathbf{P}_{2} \mathbf{T}^{(m n)} \mathbf{P}_{2} .
$$

With equation (38) we can write equation (37) as

$$
-\mathbf{P}_{2}\left(\mathbf{I}+\left(\mathbf{X}^{(m)}\right)^{T}\right) \mathbf{P}_{2} \mathbf{T}^{(m n)} \mathbf{P}_{2}=\mathbf{T}^{(m n)} \mathbf{X}^{(n)} \mathbf{P}_{2} \Longrightarrow \mathbf{X}^{(n)}=-\mathbf{T}^{(n m)} \mathbf{P}_{2}\left(\mathbf{I}+\left(\mathbf{X}^{(m)}\right)^{T}\right) \mathbf{P}_{2} \mathbf{T}^{(m n)} .
$$

where we have used the fact that

$$
\left(\mathbf{T}^{(m n)}\right)^{-1}=\mathbf{T}^{(n m)} .
$$

In particular we may take $m=0$ to obtain

$$
\mathbf{X}^{(n)}=-\mathbf{T}^{(n 0)} \mathbf{P}_{2}\left(\mathbf{I}+\left(\mathbf{X}^{(0)}\right)^{T}\right) \mathbf{P}_{2} \mathbf{T}^{(0 n)}=-\mathbf{I}-\mathbf{T}^{(n 0)} \mathbf{P}_{2}\left(\mathbf{X}^{(0)}\right)^{T} \mathbf{P}_{2} \mathbf{T}^{(0 n)} .
$$

Thus, we can express all the matrices $\mathbf{X}^{(n)}$ in terms of a single matrix $\mathbf{X}^{(0)}$. By setting $n=0$ in equation (41), we find that since $\mathbf{T}^{(00)}=\mathbf{I}, \mathbf{X}^{(0)}$ must satisfy

$$
\mathbf{X}^{(0)}+\mathbf{P}_{2}\left(\mathbf{X}^{(0)}\right)^{T} \mathbf{P}_{2}=-\mathbf{I} .
$$

From equation (42) it is easy to see that $\mathbf{X}^{(0)}$ has the following form:

$$
\mathbf{X}^{(0)}=\left(\begin{array}{cccc}
-\frac{1}{2}+\alpha & x_{12} & x_{13} & x_{14} \\
x_{21} & -\frac{1}{2}-\alpha & x_{23} & x_{24} \\
-x_{24} & x_{14} & -\frac{1}{2}+\beta & x_{34} \\
x_{23} & -x_{13} & x_{43} & -\frac{1}{2}-\beta
\end{array}\right),
$$

where $x_{i j}, \alpha$ and $\beta$ are arbitrary complex numbers. 


\section{Boundary conditions}

The boundary conditions are determined by the geometry of the system. Here we show how the boundary conditions can be imposed for a multilayer, which is infinite in the $z$-direction. A wide class of problems deal with diffusive scattering. One way to take into account the diffusive scattering is to describe it via an effective complex potential, like, for instance, a coherent potential. As a consequence the wave vectors (17) acquire a positive imaginary part [3], resulting in a vanishing GF for $\left|z-z^{\prime}\right| \rightarrow \infty$. This boundary condition implies that the GF can only contain exponentials of the form $\exp \left[\mathrm{i} k\left(z-z^{\prime}\right)\right]$ for $z>z^{\prime}$ in the right outer layer and $\exp \left[-\mathrm{i} k\left(z-z^{\prime}\right)\right]$ for $z<z^{\prime}$ in the left outer layer.

In the following we will consider the solution for $G^{\uparrow}$. The derivation of $G^{\downarrow}$ is completely equivalent. From equation (22) we see that for

$$
\mathbf{G}^{\uparrow}\left(z>z^{\prime}\right)=\Phi^{(n)}(z) \mathbf{A}^{(n)}\left(\mathbf{A}^{(0)}\right)^{-1}\left(\mathbf{I}+\mathbf{X}^{(0)}\right)\left(\Phi^{(0)}\left(z^{\prime}\right)\right)_{2}^{-1}
$$

to meet this condition $\left(\mathbf{I}+\mathbf{X}^{(0)}\right)$ must have the second and fourth columns zero, i.e. $\mathbf{X}^{(0)}$ must have the form

$$
\mathbf{X}^{(0)}=\left(\begin{array}{cccc}
0 & 0 & 0 & 0 \\
x_{21} & -1 & x_{23} & 0 \\
0 & 0 & 0 & 0 \\
x_{23} & 0 & x_{43} & -1
\end{array}\right),
$$

where we have already accounted for the symmetry conditions set out by equation (43). Therefore, we have the three parameters $x_{21}, x_{23}, x_{43}$ to impose boundary conditions on the right end of the multilayer.

So far we have only used the $\boldsymbol{\Phi}$ basis. Now we wish to express the Green function through the $\mathbf{F}$ basis. It is easy to see that

$$
\left(\Phi^{(m)}(z)\right)^{-1}=\mathbf{A}^{(m)}\left(\mathbf{F}_{u p}^{(m)}(z)\right)^{-1},
$$

and thus

$$
\mathbf{h}^{(m)}\left(z^{\prime}\right)=\left(\mathbf{A}^{(m)}\right)^{-1} \mathbf{X}^{(m)}\left(\Phi^{(m)}\left(z^{\prime}\right)\right)_{2}^{-1}=\left(\mathbf{A}^{(m)}\right)^{-1} \mathbf{X}^{(m)} \mathbf{A}^{(m)}\left(\mathbf{F}_{u p}^{(m)}\left(z^{\prime}\right)\right)_{2}^{-1} .
$$

Therefore, we can write

$$
\begin{aligned}
\mathbf{G}^{\uparrow}\left(z>z^{\prime}\right) & =\mathbf{F}_{u p}^{(n)}(z)\left(\left(\mathbf{F}_{u p}^{(m)}\left(z^{\prime}\right)\right)_{2}^{-1}+\mathbf{h}^{(m)}\left(z^{\prime}\right)\right) \\
& =\mathbf{F}_{u p}^{(n)}(z)\left(\left(\mathbf{F}_{u p}^{(m)}\left(z^{\prime}\right)\right)_{2}^{-1}+\left(\mathbf{A}^{(m)}\right)^{-1} \mathbf{X}^{(m)} \mathbf{A}^{(m)}\left(\mathbf{F}_{u p}^{(m)}\left(z^{\prime}\right)\right)_{2}^{-1}\right) \\
& =\mathbf{F}_{u p}^{(n)}(z)\left(\mathbf{I}+\left(\mathbf{A}^{(m)}\right)^{-1} \mathbf{X}^{(m)} \mathbf{A}^{(m)}\right)\left(\mathbf{F}_{u p}^{(m)}\left(z^{\prime}\right)\right)_{2}^{-1} \\
& =\mathbf{F}_{u p}^{(n)}(z)\left(-\left(\mathbf{A}^{(0)}\right)^{-1} \mathbf{P}_{2}\left(\mathbf{X}^{(0)}\right)^{T} \mathbf{P}_{2} \mathbf{A}^{(0)}\right)\left(\mathbf{F}_{u p}^{(m)}\left(z^{\prime}\right)\right)_{2}^{-1},
\end{aligned}
$$

where we have used equation (41) and definition (32) in the last line.

Now we assume a special form for the fundamental matrix $\mathbf{F}^{(N)}$. Note that equation (45) for the matrix $\mathbf{X}^{(0)}$ was obtained without any assumption concerning the matrix $\mathbf{A}^{(0)}$ and consequently we are free in the choice of $\mathbf{F}^{(N)}$. We demand that $\mathbf{F}^{(N)}$ be identical to $\boldsymbol{\Phi}^{(N)}(z)$. In this case, as is seen from equation (48), the proper boundary conditions are justified if the matrix

$$
\mathbf{V} \equiv \mathbf{A}^{(0)-1} \mathbf{L}_{2} \mathbf{X}^{(0) T} \mathbf{L}_{2} \mathbf{A}^{(0)}
$$

has the following form:

$$
\mathbf{V}=\left(\begin{array}{cccc}
V_{11} & V_{12} & V_{13} & V_{14} \\
0 & 0 & 0 & 0 \\
V_{31} & V_{32} & V_{33} & V_{34} \\
0 & 0 & 0 & 0
\end{array}\right)
$$


Solving the matrix equation (49) we find that

and

$$
\mathbf{V}=\left(\begin{array}{cccc}
-1 & V_{12} & 0 & V_{14} \\
0 & 0 & 0 & 0 \\
0 & V_{32} & -1 & V_{34} \\
0 & 0 & 0 & 0
\end{array}\right)
$$

$$
\mathbf{A}^{(0)}=\left(\begin{array}{cccc}
a_{11} & -a_{11} V_{12}-a_{13} V_{32} & a_{13} & -a_{11} V_{14}-a_{13} V_{34} \\
a_{31} x_{23}+a_{11} x_{21} & a_{22} & a_{33} x_{23}+a_{13} x_{21} & a_{24} \\
a_{31} & -a_{31} V_{12}-a_{33} V_{32} & a_{33} & -a_{31} V_{14}-a_{33} V_{32} \\
a_{11} x_{23}+a_{31} x_{43} & a_{42} & a_{13} x_{23}+a_{33} x_{43} & a_{44}
\end{array}\right)
$$

The matrix $\mathbf{A}^{(0)}$ ensures the correct boundary conditions for $z \rightarrow \infty$. This specific form of $\mathbf{A}^{(0)}$ must be compatible with our choice of basic functions (16). Our choice $\mathbf{F}^{(N)}(z)=\boldsymbol{\Phi}^{(N)}(z)$ requires that $\mathbf{A}^{(N)}$ be the identity matrix. Then the relation

$$
\mathbf{T}^{(0, N)} \mathbf{A}^{(N)}=\mathbf{A}^{(0)}
$$

gives a set of equations for the matrix elements of $\mathbf{A}(0)$ which we consider below.

Taking the product of $\mathbf{T}^{(0, N)}$ and the first and the third columns of the matrix $\mathbf{A}^{(N)}$ in equation (53), we obtain

$$
\mathbf{T}^{(0, N)}\left(\begin{array}{l}
1 \\
0 \\
0 \\
0
\end{array}\right)=\left(\begin{array}{c}
a_{11} \\
a_{31} x_{23}+a_{11} x_{21} \\
a_{31} \\
a_{11} x_{23}+a_{31} x_{43}
\end{array}\right)
$$

and

$$
\mathbf{T}^{(0, N)}\left(\begin{array}{l}
0 \\
0 \\
1 \\
0
\end{array}\right)=\left(\begin{array}{c}
a_{13} \\
a_{33} x_{23}+a_{13} x_{21} \\
a_{33} \\
a_{13} x_{23}+a_{33} x_{43}
\end{array}\right)
$$

The first and third lines in equations (54) and (55) imply that

$$
a_{11}=T_{11}, \quad a_{31}=T_{31}, \quad a_{13}=T_{13}, \quad a_{33}=T_{33} .
$$

where a superscript of $(0, N)$ is implied on all the $T_{i j}$. The second and fourth lines in equations (54) and (55) form a system of linear equations:

$$
\begin{array}{ll}
x_{23} T_{31}+x_{21} T_{11}=T_{21}, & x_{23} T_{11}+x_{43} T_{31}=T_{41} \\
x_{23} T_{33}+x_{21} T_{13}=T_{23}, & x_{23} T_{13}+x_{43} T_{33}=T_{43}
\end{array}
$$

From equation (57), we can determine the unknown elements of the matrix $\mathbf{X}^{(0)}$. The first three can be solved explicitly giving

$$
\begin{aligned}
& x_{43}=\frac{T_{11} T_{13} T_{21}-\left(T_{11}\right)^{2} T_{23}-T_{13} T_{31} T_{41}+T_{11} T_{33} T_{41}}{-\left(T_{31}\right)^{2} T_{13}+T_{11} T_{31} T_{33}}, \\
& x_{21}=\frac{T_{23} T_{31}-T_{21} T_{33}}{T_{13} T_{31}-T_{11} T_{33}}, \quad x_{23}=\frac{T_{13} T_{21}-T_{11} T_{23}}{T_{13} T_{31}-T_{11} T_{33}} .
\end{aligned}
$$


The last equation in (57) forces a condition of compatibility for the solutions in (58):

$$
-T_{13} T_{21}+T_{23} T_{11}-T_{33} T_{41}+T_{43} T_{31}=0,
$$

where we have used equations (27) and (32) to simplify the expression.

Now we need to find the matrix elements of $\mathbf{V}$. Taking a product of $\mathbf{T}^{(0, N)}$ and the second and the fourth columns of $\mathbf{A}^{(N)}$ in equation (53) we find

$$
\mathbf{T}^{(0 N)}\left(\begin{array}{l}
0 \\
1 \\
0 \\
0
\end{array}\right)=\left(\begin{array}{c}
-T_{11} V_{12}-T_{13} V_{32} \\
a_{22} \\
-T_{31} V_{12}-T_{33} V_{32} \\
a_{42}
\end{array}\right),
$$

and

$$
\mathbf{T}^{(0 N)}\left(\begin{array}{l}
0 \\
0 \\
0 \\
1
\end{array}\right)=\left(\begin{array}{c}
-T_{11} V_{14}-T_{13} V_{34} \\
a_{24} \\
-T_{31} V_{14}-T_{33} V_{32} \\
a_{44}
\end{array}\right) .
$$

Using these equations we obtain

$$
\left(\begin{array}{l}
V_{12} \\
V_{32}
\end{array}\right)=\frac{1}{T_{11} T_{33}-T_{13} T_{31}}\left(\begin{array}{l}
T_{32} T_{13}-T_{12} T_{33} \\
T_{12} T_{31}-T_{11} T_{32}
\end{array}\right)
$$

and

$$
\left(\begin{array}{l}
V_{14} \\
V_{34}
\end{array}\right)=\frac{1}{T_{11} T_{33}-T_{13} T_{31}}\left(\begin{array}{l}
T_{13} T_{34}-T_{14} T_{33} \\
T_{14} T_{31}-T_{11} T_{34}
\end{array}\right)
$$

Thus, we have constructed the matrices $\mathbf{A}^{(0)}, \mathbf{V}$ and $\mathbf{X}^{(0)}$ and thereby solved the problem.

\section{Conclusion}

We have proposed a constructive way to build a symmetric one-electron GF for the magnetic multilayer with magnetization in the plane of the layers. To build this GF we solved the following problems: finding the solution of the systems of differential equations (7) and (8) of general form; matching the solutions on the interfaces; imposing proper boundary conditions and employing free parameters to get a symmetric function with respect to permutation of the variables $z \rightarrow z^{\prime}$ and $\sigma \rightarrow \sigma^{\prime}$. Our consideration is valid for complex wave vectors (17) which allows for extending this method to systems with insulating layers. Also our consideration can be applied to systems with finite lateral dimensions like magnetic nanowires [12].

Note that the described matching procedure, as well as the procedure providing for the boundary conditions, can be applied for a layer potential which differs from the usual rectangular potential. In contrast, the possibility of imposing some symmetry condition requires additional investigation. For our consideration the equalities (27) and (28) were essential. The validity of these equalities is based upon the specific form of basic functions.

The proposed GF is useful for the investigation of electronic transport properties of magnetic multilayers. Though our consideration was performed for specific boundary conditions which are typical for current-perpendicular-to-plane geometry, the proposed approach can be applied to a multilayer of finite transverse dimension with zero boundary conditions on the outer interfaces.

\section{Acknowledgments}

This work was supported by W. M. Keck Foundation, Seagate Research, and the Nebraska Research Initiative. 


\section{References}

[1] Tsymbal E Y and Pettifor D G 2001 Perspectives of giant magnetoresistance Solid State Physics vol 56 ed H Ehrenreich and F Spaepen (San Diego, CA: Academic) pp 113

Tsymbal E Y and Pettifor D G 1996 Phys. Rev. B 5415314

[2] Tsymbal E Y, Mryasov O N and LeClair P R 2003 J. Phys.: Condens. Matter 15 R109

[3] Vedyayev A, Dieny B and Ryzhanova N 1992 Europhys. Lett. 19329

[4] Camblong H E, Levy P M and Zhang S 1995 Phys. Rev. B 5116052

[5] Tagirov L 1999 Phys. Rev. Lett. 832058

[6] Burton J D, Kashyap A, ZhuravlevMYe, Skomski R, Tsymbal E Y, Jaswal S S, Mryasov O N and Chantrell RW 2003 Appl. Phys. Lett. 85251

[7] Zhuravlev M Ye, Tsymbal E Y and Vedyayev A V 2005 Phys. Rev. Lett. 94026806

[8] Garcia-Moliner F and Velasco V R 1992 Theory of Single and Multiple Interfaces (Singapore: World Scientific)

[9] Vedyayev A, Dieny B, Ryzhanova N, Genin J B and Cowcache C 1994 Europhys. Lett. 25465

[10] Zhuravlev M Ye, Lutz H O and Vedyayev A V 2001 J. Phys. A: Math. Gen. 341

[11] Perez-Alvarez R, Garcia-Moliner F and Velasco V R 1995 J. Phys.: Condens. Matter 7 2037

[12] Zhuravlev M Ye, Tsymbal E Y, Jaswal S S, Vedyayev A V and Dieny B 2003 Appl. Phys. Lett. 833534 